\title{
Collective-Mode Enhanced Matter-Wave Optics
}

\author{
Christian Deppner®, ${ }^{1}$ Waldemar Herr, ${ }^{1,2}$ Merle Cornelius, ${ }^{3}$ Peter Stromberger, ${ }^{4}$ Tammo Sternke, ${ }^{3}$ \\ Christoph Grzeschik, ${ }^{5}$ Alexander Grote, ${ }^{4}$ Jan Rudolph $\odot,{ }^{1,}{ }^{*}$ Sven Herrmann $\odot{ }^{3}$ Markus Krutzik, \\ André Wenzlawski, ${ }^{4}$ Robin Corgier, ${ }^{1,6, \dagger}$ Eric Charron $\odot,{ }^{6}$ David Guéry-Odelin, ${ }^{7}$ Naceur Gaaloul $\odot,{ }^{1}$ \\ Claus Lämmerzahl@ ${ }^{3}$ Achim Peters, ${ }^{5}$ Patrick Windpassinger, ${ }^{4}$ and Ernst M. Rasel ${ }^{1, \sharp}$ \\ ${ }^{1}$ Institut für Quantenoptik, Leibniz Universität Hannover, Welfengarten 1, D-30167 Hannover, Germany \\ ${ }^{2}$ Deutsches Zentrum für Luft- und Raumfahrt e.V., Institut für Satellitengeodäsie und Inertialsensorik, \\ c/o Leibniz Universität Hannover, DLR-SI, Callinstraße 36, D-30167 Hannover, Germany \\ ${ }^{3}$ ZARM, Universität Bremen, Am Fallturm 2, D-28359 Bremen, Germany \\ ${ }^{4}$ Johannes Gutenberg-Universität Mainz, Staudingerweg 7, D-55128 Mainz, Germany \\ ${ }^{5}$ Institut für Physik, Humboldt-Universität zu Berlin, Newtonstraße 15, D-12489 Berlin, Germany \\ ${ }^{6}$ Université Paris-Saclay, CNRS, Institut des Sciences Moléculaires d'Orsay, F-91405 Orsay, France \\ ${ }^{7}$ Laboratoire de Collisions Agrégats Réactivité, CNRS, IRSAMC, Université de Toulouse, \\ 118 Route de Narbonne, F-31062 Toulouse, France
}

(Received 13 November 2020; revised 14 June 2021; accepted 25 June 2021; published 30 August 2021)

In contrast to light, matter-wave optics of quantum gases deals with interactions even in free space and for ensembles comprising millions of atoms. We exploit these interactions in a quantum degenerate gas as an adjustable lens for coherent atom optics. By combining an interaction-driven quadrupole-mode excitation of a Bose-Einstein condensate (BEC) with a magnetic lens, we form a time-domain matter-wave lens system. The focus is tuned by the strength of the lensing potential and the oscillatory phase of the quadrupole mode. By placing the focus at infinity, we lower the total internal kinetic energy of a BEC comprising 101(37) thousand atoms in three dimensions to $3 / 2 k_{B} \cdot 38_{-7}^{+6} \mathrm{pK}$. Our method paves the way for free-fall experiments lasting ten or more seconds as envisioned for tests of fundamental physics and high-precision BEC interferometry, as well as opens up a new kinetic energy regime.

DOI: 10.1103/PhysRevLett.127.100401

Optics with matter waves shares many analogies with its counterpart for light. However, matter can interact via electromagnetic forces: a well known fact in electron or ion optics, where the Coulomb repulsion causes particle beams to diverge, deteriorating their quality [1]. Similarly, interactions accelerate the expansion of a repulsive quantum gas in free fall and, moreover, become dominant at ultralow temperatures, setting a lower limit to the internal kinetic energy of the gas [2].

So far, evaporative cooling [3] and spin gradient cooling [4] permitted to reach three-dimensional internal kinetic energies below 500 and $350 \mathrm{pK}$, respectively. In terms of effective temperatures, employing matter-wave lenses based on magnetic [5-7], electrostatic [8], or optical [9] forces made it possible to reduce the internal kinetic energy of a BEC to about $50 \mathrm{pK}$ [10], albeit only in two dimensions.

We tailor the expansion of a ${ }^{87} \mathrm{Rb} \mathrm{BEC}$ by exploiting a collective-mode excitation in the BEC [11,12] in combination with a magnetic lens. Both act together like a timedomain matter-wave lens system for all three spatial dimensions. The focus of the lens system can be tuned by releasing the BEC at an appropriate phase of the collective-mode oscillation and the strength of the lensing potential. When focusing at infinity, we achieve a total internal kinetic energy in three dimensions of as low as $3 / 2 k_{B} \cdot 38_{-7}^{+6} \mathrm{pK}$.

Such atomic ensembles allow for placing better experimental constraints on proposed modifications of quantum theory [13-15], predicting tiny deviations from the standard expansion of a quantum gas. Moreover, for the first time, they fulfill the strict requirements imposed by atom interferometers exploiting free-fall times of tens of seconds [16-18] as needed, e. g. for a stringent quantum test of the equivalence principle [19-21], gravitational wave detection $[22,23]$, or the determination of the gravitational constant [24] and the photon recoil [25]. In these precision experiments, the residual motion of the atoms couples to rotations [26-28] or to wave-front distortions of the interferometry light beam [29-31], leading to a phase noise or bias in the interferometer. Additionally, the atomic expansion limits the efficiency of large momentum beam splitters proposed for high-precision experiments [32-35].

Our matter-wave lens system is implemented using an atom chip [Fig. 1(a)], which permits us to excite the BEC to perform collective-mode oscillations [Fig. 1(b)] to release it at a specific phase and to shape it with a magnetic lens. Figures 1(c)-1(e) compare the simulated absorption images 
(a) experimental setup

(b) interaction-driven quadrupole-mode oscillation

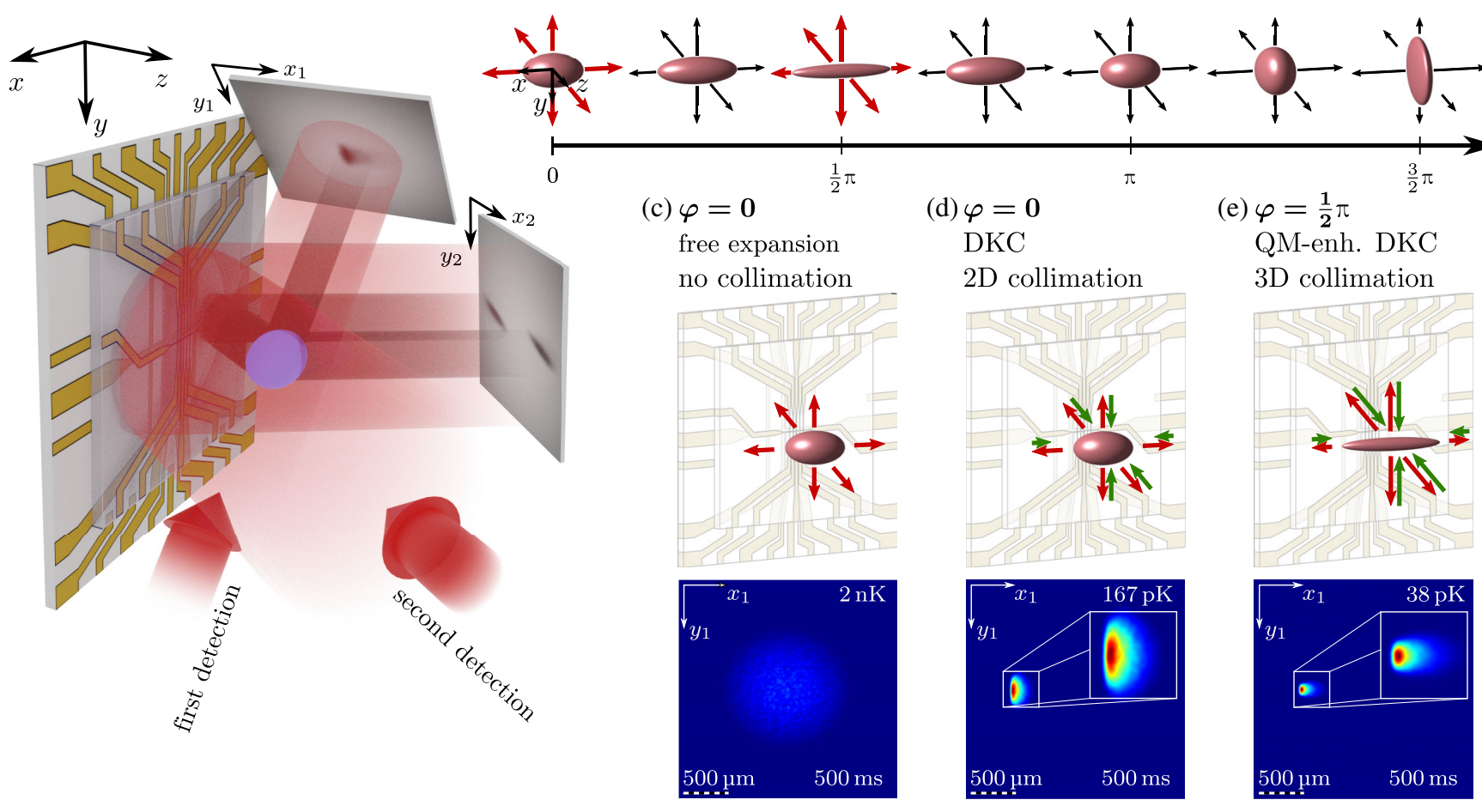

FIG. 1. Interaction based time-domain matter-wave lens system. Bose-Einstein condensate (BEC), created on an atom chip, is released and then either freely drifting or briefly exposed to a magnetic lensing potential. Absorption images from two different directions provide full three-dimensional (3D) information of the BEC's position and spatial distribution, as shown in Fig. 1(a). Before its release, BEC is excited to perform quadrupole-mode oscillation as depicted in Fig. 1(b). Depending on its phase $\varphi$, the size and expansion of the BEC varies. In Fig. 1(c), the BEC freely expands in all directions with an internal kinetic energy of $3 / 2 k_{B} \cdot 2 \mathrm{nK}$. In Fig. 1(d), green arrows depict the effect of a cylindrical magnetic lens, reducing $U_{\text {kin }}$ to $3 / 2 k_{B} \cdot 167 \mathrm{pK}$. Choosing release at an oscillatory phase, such that the BEC is only weakly expanding in the axial direction, together with applying a cylindrical magnetic lens, allows for reaching the lowest energy of $3 / 2 k_{B} \cdot 38 \mathrm{pK}$, as shown in Fig. 1(e).

of a BEC obtained with and without our method. The release of the BEC at a phase $\varphi$ of the oscillation, where it expands in all three directions (red arrows) without and with application of a magnetic lens (green arrows), leading to delta-kick collimation (DKC) in Figs. 1(c) and 1(d), serves as a reference for our experiments. Without the magnetic lens, we achieve an internal kinetic energy of $3 / 2 k_{B} \cdot 2 \mathrm{nK}$. The atom-chip based magnetic lens, which typically has a cylindrical shape, can lower the expansion rate mainly along the radial direction, resulting in a total energy of $3 / 2 k_{B} \cdot 167 \mathrm{pK}$. Exploiting the quadrupole-mode (QM) oscillation, we lower the BEC's expansion along the axial direction, where the magnetic lens lacks refractive power. By tailoring the highly anisotropic expansion to the magnetic lens, our time-domain lens system resulted in an internal kinetic energy of $3 / 2 k_{B} \cdot 38 \mathrm{pK}$, as shown in Fig. 1(e).

The experiments, using the high-flux BEC source detailed in [36], were conducted in the Bremen Drop Tower, providing a free fall lasting for $4.74 \mathrm{~s}$. During the drops, BECs of about 100 thousand atoms were created in a cylindrically shaped Ioffe-Pritchard trap $[37,38]$ with final evaporation-trap frequencies of $f_{\text {evap }}=\{24,457,462\} \mathrm{Hz}$ along the $x, y$, and $z$ directions, respectively. Before the release, we excite a quadrupolemode oscillation in the BEC $[11,12]$ by swiftly reducing the magnetic bias field in $x$ direction within $0.5 \mathrm{~ms}$, increasing only the strong trap frequencies to $f_{\mathrm{QM}}=\{24,550,554\} \mathrm{Hz}$. After a delay of $0.4 \mathrm{~ms}$, accounting for a magnetic field settling time, the BEC is transported along the $z$ direction within $150 \mathrm{~ms}$ to its release position $1462 \mu \mathrm{m}$ away from the atom chip by reducing the magnetic bias field in the $y$ direction. During this transport, the trap frequencies reduce to $f_{\text {release }}=\{9.1,27.9,24.6\} \mathrm{Hz}$. A shortcut-to-adiabaticity protocol is used to minimize the amplitude of a residual center-of-mass dipole oscillation of the BEC to $A_{\text {release }}=$ $\{1.20(59), 0.30(12), 4.00(19)\} \mu \mathrm{m}[39]$.

During the free expansion of the BECs, lasting $80 \mathrm{~ms}$, internal interaction energy is converted into kinetic energy and the ensemble reaches the ballistic regime. Hereafter the three-dimensional predominantly cylindrical magnetic lens is applied (TOF $=0 \mathrm{~ms}$; see also Fig. 3). Close to its center, the lens potential along the $x$ and $y$ axes is well described by the harmonic approximation with potential frequencies of $\omega_{x}=2 \pi \cdot 2.9 \mathrm{rad} / \mathrm{s}$ and 
$\omega_{y}=2 \pi \cdot 10.6 \mathrm{rad} / \mathrm{s}$. However, along the $z$ axis, the potential deviates from a simple harmonic shape at larger distances to the center and can be approximated by $V_{z}=$ $1 / 2 m \omega_{z}^{2} z^{2}\left(1+z / L 3+z^{2} / L 4^{2}\right)$, with $\omega_{z}=2 \pi \cdot 10.6 \mathrm{rad} / \mathrm{s}$, $L 3=1225 \mu \mathrm{m}$, and $L 4=2933 \mu \mathrm{m}$. After a $2.42 \mathrm{~ms}$ exposure to the lens, a subsequent radio-frequency driven adiabatic-rapid-passage [40] transfers $87.1(2) \%$ of the atoms from the state of $\left|F=2, m_{F}=2\right\rangle$ into $\left|F=2, m_{F}=0\right\rangle$ in order to reduce their susceptibility to residual magnetic fields [41].

Absorption images [42] from one of the two directions available [Fig. 1(a)] taken at different times provide complete information about the motion and expansion of the BEC over time. Without applying our time-domain lens system, BECs were imaged up to $160 \mathrm{~ms}$ after release. With our lens system, we could stretch this time to $2 \mathrm{~s}$. To record these data, we performed 56 BEC experiments over a time of ten weeks, consuming 34 drops in the Bremen Drop Tower. Despite this limited number of drops, the data are sufficient to gauge our simulation of the experiment and to optimize our matter-wave lens system.

Lowering the internal kinetic energy of the ensemble requires an analysis of the excited quadrupole mode employed in our method. For this purpose, we determined the aspect ratios of the Thomas-Fermi radii $R$ by absorption imaging for varying hold times of the BEC in the release trap. In Fig. 2, the measured aspect ratios, defined by $R_{x_{1}} / R_{y_{1}}$ (blue) and $R_{x_{2}} / R_{y_{2}}$ (orange), are shown together with the results of a $3 \mathrm{D}$ collective-mode simulation of the BEC in the magnetic trap (solid lines) based on a variational approach [43-45]. A damping time of $300 \mathrm{~ms}$ was introduced in our simulation to consider residual thermal atoms in the trap [46,47], as well as the anharmonicities of the lens potential [48]. The Fourier transform of the shape oscillation shows a large amplitude of the quadrupole mode with a small admixture of a monopole mode.

A hold time of $18.46 \mathrm{~ms}$ (dashed line in Fig. 2) turned out to be optimal for our purpose because the BEC had already passed the turning point of the quadrupole-mode oscillation along the $x$ direction. The collapse along the weak axis counteracts the emerging accelerating expansion due to the interaction energy, improving the collimation along this axis. This hold time coincides with the turning point of the dipole oscillation in the $z$ direction, reducing the center-of-mass velocity of the BEC in total to $v_{x y z}=\{56(26),-56(69), 139(17)\} \mu \mathrm{m} / \mathrm{s}$.

Figure 3 summarizes the experimental results (colored diamonds) as well as the numerical simulation (colored lines) of tailoring the expansion of the BEC with our timedomain lens system by focusing the matter wave at infinity in 3D. For comparison, the effect of the oscillation without a magnetic lens (circles) as well as numerical simulations employing only a magnetic lens for collimation (dashed colored lines) are shown.
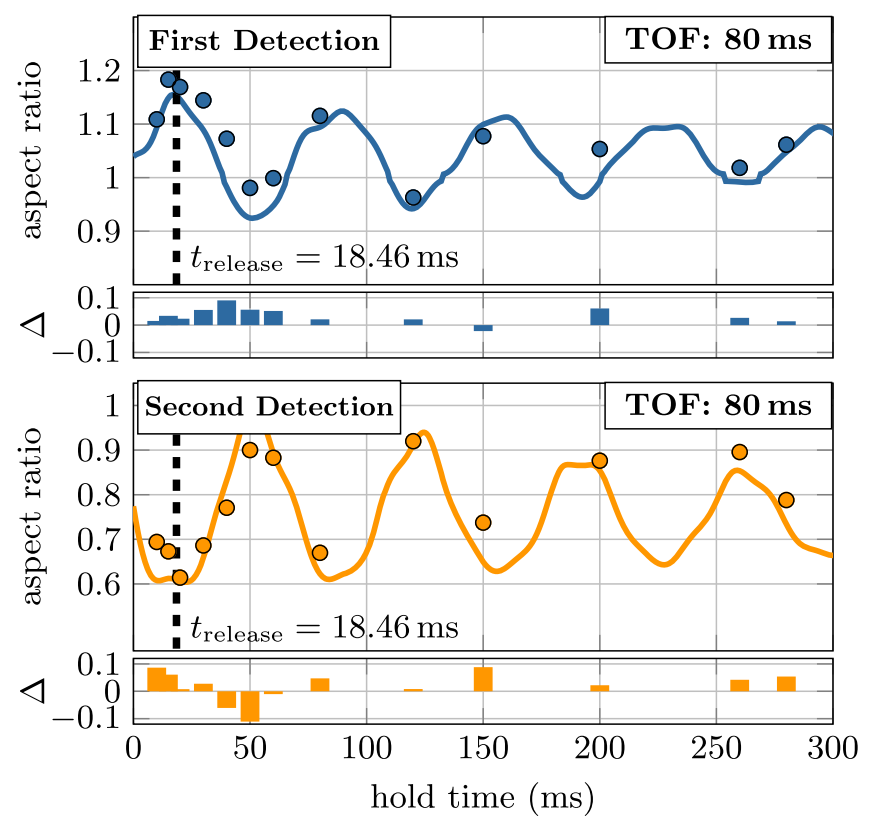

FIG. 2. Collective-mode oscillation of BEC in release trap as observed after $80 \mathrm{~ms}$ time of flight. Aspect ratio in dependence of hold time in release trap derived from both detection systems (circles) shown next to our simulation (lines). Bar graphs show their residuals to simulation. Dashed lines indicate instance of release where our time-domain lens system is optimally collimating the BEC.

The root of the second moment of the one-dimensional density distribution integrated along the orthogonal direction serves as a measure of the BEC's size. This is motivated by the shapes of the BECs observed after evolution times of several hundreds of milliseconds. Here, a more and more pronounced tail appears in the density distribution, pointing in the $z$ direction, which stems from the anharmonicity of the magnetic lens. Therefore, the shape of the BEC along this direction cannot be characterized by a Thomas-Fermi fit, and a simple harmonic scaling approach [49] is not sufficient to describe its evolution.

In order to obtain numerical pictures, we model the spatial density and corresponding velocity distribution of the BEC after a free expansion of $80 \mathrm{~ms}$. The effect of the lens and the free evolution until detection is calculated based on a three-dimensional interaction-free particle simulation, where the atoms as classical particles are stochastically sampled from a classical probability distribution that gives the correct position and momentum densities. From the spatial densities, absorption images for all directions of observation are computed. To fit the numerical images to the experimental data, the pixelwise difference is computed and minimized at once using a nonlinear least-squares algorithm. Free fit parameters are global corrections to the initial spatial and velocity distributions; the effective duration of the applied lens, accounting for a noninstantaneous switching; as well as 


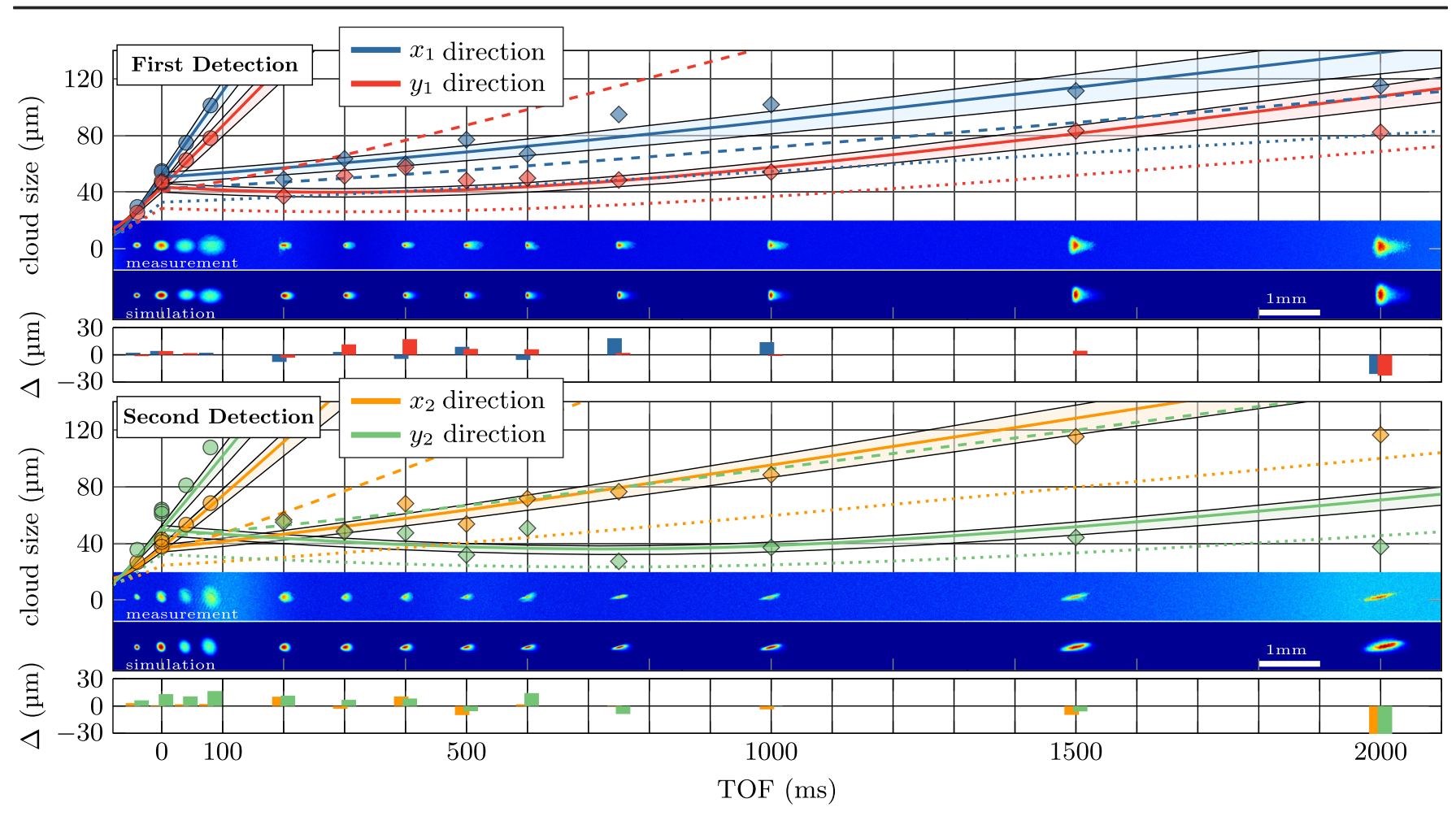

FIG. 3. Free evolution of BEC collimated with our time-domain lens system. As a measure of size, the root of the one-dimensional second moments of the absorption images' spatial distribution depicted underneath the respective graphs is shown. Results obtained employing only quadrupole-mode oscillation (circles; compare Table I "Free exp.") and impact of time-domain lens system (diamonds; compare Table I "QM-enh. DKC") shown next to our 3D particle simulation (colored lines) fitted to experimental data. Bar graphs represent their residuals to 3D particle simulation. Shaded areas reflect uncertainty in particle number. BECs featured typically 101(37) thousand atoms and internal kinetic energy of $3 / 2 k_{B} \cdot 38_{-7}^{+6} \mathrm{pK}$ after application of our time-domain lens system. Our simulation allows us to compare our method with delta-kick collimation only, which results in a significantly larger expansion rate (dashed lines; compare Table I "DKC"). Additionally, dotted lines compare simulated expansion of a BEC with 10 thousand atoms displaying a lower meanfield energy, corresponding to $U_{\text {kin }}=3 / 2 k_{B} \cdot 14 \mathrm{pK}$.

a rotation and position offset of the lens potential relative to the BEC. Furthermore, the efficiency of the adiabatic rapid passage of $87.1(2) \%$ is taken into account. Shot-to-shot atom fluctuation and frequency instability of the detection laser, which affect each data point individually, required additional correction parameters to be added. A more detailed description of the fitting procedure can be found in the Supplemental Material [45].

From the fit, all relevant properties (especially the velocity distribution) can be obtained. As a cross-check, a particle number of 101(37) thousand atoms was fitted, which is in good agreement with the particle number of 98(38) thousand atoms extracted from five experiments, where a BEC is simply released from the trap and imaged after $80 \mathrm{~ms}$ of free evolution. The spread in particle number arises due to day-to-day fluctuations of the system's performance and has the largest influence on the BEC's expansion and, by this, on the uncertainty of the total internal kinetic energy. The combined uncertainties of the other fit parameters contribute on the order of less than $1 \mathrm{pK}$ and are therefore negligible.
From the numerical images (Fig. 3; "simulation"), the expansion of the BEC (colored lines) and the fit uncertainties (shaded areas) were computed. The timedomain lens system reduces the total internal kinetic energy

TABLE I. One-dimensional (1D) and 3D internal kinetic energy of BEC during free expansion (Free exp.) and after DKC with and without quadrupole-mode enhancement (QM-enh.). 1D internal kinetic energy in, e. g., $x$ direction defined via second moment of twice integrated 3D-velocity distribution $f(v)$ via $U_{\mathrm{kin}, x}=$ $1 / 2 k_{B} T_{x}=1 / 2 m_{\mathrm{Rb}} \sigma_{v, x}^{2} \quad$ with $\quad \sigma_{v, x}^{2}=\int \mathrm{d} v_{x} v_{x}^{2} \cdot \iint \mathrm{d} v_{y} \mathrm{~d} v_{z} f\left(v_{x y z}\right)$. Residual interaction energy of BEC constitutes limit for lowest achievable kinetic energy in this setup.

\begin{tabular}{lcrcc}
\hline \hline Dimension & $\begin{array}{c}\text { Free exp. } \\
(\mathrm{pK})\end{array}$ & $\begin{array}{c}\text { DKC } \\
(\mathrm{pK})\end{array}$ & $\begin{array}{c}\text { QM-enh. DKC } \\
(\mathrm{pK})\end{array}$ & $\begin{array}{c}\text { Limit } \\
(\mathrm{pK})\end{array}$ \\
\hline$U_{\mathrm{kin}, x} /\left(1 / 2 k_{B}\right)$ & 659 & 447 & $62_{-11}^{+8}$ & 45 \\
$U_{\text {kin }, y} /\left(1 / 2 k_{B}\right)$ & 3020 & 36 & $24_{-4}^{+3}$ & 17 \\
$U_{\text {kin }, z} /\left(1 / 2 k_{B}\right)$ & 2658 & 16 & $29_{-7}^{+6}$ & 16 \\
$U_{\text {kin }} /\left(3 / 2 k_{B}\right)$ & 2112 & 167 & $38_{-7}^{+6}$ & 26 \\
\hline \hline
\end{tabular}


of the initial BECs, amounting to $3 / 2 k_{B} \cdot 2 \mathrm{nK}$ to $3 / 2 k_{B} \cdot 38_{-7}^{+6} \mathrm{pK}$, corresponding to an expansion velocity of as low as $\sigma_{v}=\left\{77_{-5}^{+7}, 47_{-3}^{+4}, 53_{-6}^{+7}\right\} \mu \mathrm{m} / \mathrm{s}$. The combined action of quadrupole-mode oscillation and delta-kick collimation favorably compares to the case where the delta-kick collimation is solely applied (dashed lines), leading to $U_{\text {kin }}=3 / 2 k_{B} \cdot 167 \mathrm{pK}$. Table I compares the internal kinetic energies in all three directions obtained with the different methods.

The minimal achievable internal kinetic energy is determined by the residual interaction energy of the BEC after the magnetic lens and amounts to $3 / 2 k_{B} \cdot 26 \mathrm{pK}$. Ideally, further reduction of $U_{\text {kin }}$ could be achieved by extending the time of free expansion prior to the magnetic lens, but the anharmonicities of the latter would hinder reaching even lower expansion rates in our current setup. To mitigate this lens abberation, one could form a more complex multilens system in analogy to light optics.

Even slower expansion could also be achieved by simply reducing the number of atoms. For reference, the dotted lines in Fig. 3 show the simulated expansion of a BEC with only 10 thousand atoms, leading to $U_{\text {kin }}=3 / 2 k_{B} \cdot 14 \mathrm{pK}$. However, the BEC's detectability over time decreases drastically with the atom number.

Our method allows us to largely extend the free evolution time of the BEC before it becomes too diluted to be detected by absorption imaging. Here, we define this time to be the moment the absorption signal of the BEC's peak density approaches the single-shot detection noise, which is obtained from the background noise in the absorption images.

The peak density has been simulated for a BEC shaped by our matter-wave lens system and for a simple release. Whereas, in the latter case, the BEC is visible until approximately $2.25 \mathrm{~s}$, our method extends this time to 17 s, as shown in Fig. 4. The gray line, in comparison, represents the detectability of a BEC with only 10 thousand atoms at $U_{\text {kin }}=3 / 2 k_{B} \cdot 14 \mathrm{pK}$ that, even if the internal kinetic energy is lower, is only visible for $3.5 \mathrm{~s}$.

In conclusion, interactions that are often compromising matter-wave optics were exploited using collective-mode excitations of a BEC for shaping its evolution. In combination with a magnetic lens, we realized a time-domain lens system with a focus adjustable by the oscillatory phase at the condensate's release and the strength of the lensing potential. Focusing at infinity, we reduced the free expansion of a BEC in all three dimensions, yielding unprecedentedly low internal kinetic energies of $3 / 2 k_{B} \cdot 38_{-7}^{+6} \mathrm{pK}$. In this way, we sampled the time evolution of BECs comprising 101(37) thousand atoms for up to $2 \mathrm{~s}$ during free fall in the Bremen Drop Tower.

According to our simulations, these slowly expanding BECs would be detectable by absorption imaging even after $17 \mathrm{~s}$, exceeding by far the microgravity time offered

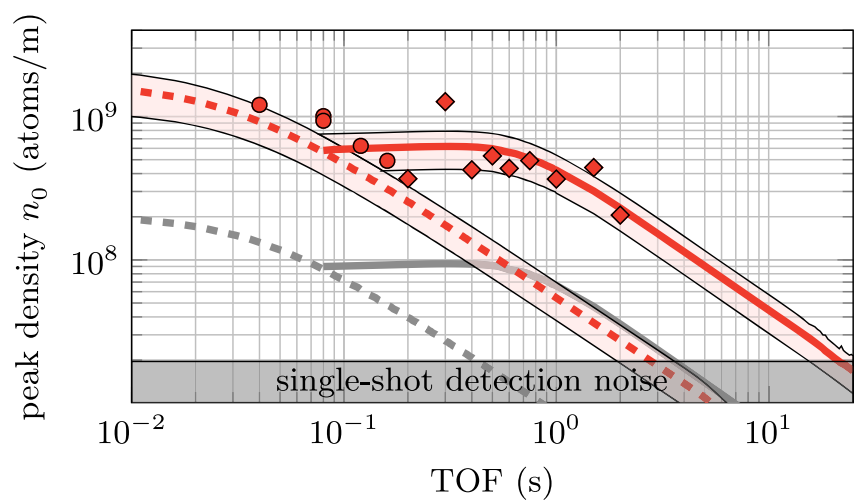

FIG. 4. Detectability of BEC using our matter-wave lens system in dependence of time of flight without (with) employing our time-domain lens system depicted as circles (diamonds). Peak density along $y_{1}$ direction of twice integrated simulated BEC's spatial density distribution compared with data and noise of our imaging system. Our freely evolving BEC with 101(37) thousand atoms at $3 / 2 k_{B} \cdot 2 \mathrm{nK}$ (dashed red line) is visible for approximately $2.25 \mathrm{~s}$, which is greatly extended to $17 \mathrm{~s}$ by reducing $U_{\text {kin }}$ to $3 / 2 k_{B} \cdot 38_{-7}^{+6} \mathrm{pK}$ using our lens system (solid red line). Shaded area reflects uncertainty in atom number. Gray lines show same simulation but with BEC composed of 10 thousand atoms leading to a much shorter detectability of $3.5 \mathrm{~s}$, although internal kinetic energy would be at $3 / 2 k_{B} \cdot 14 \mathrm{pK}$.

by the Drop Tower. They represent an exceptional input state for atom interferometry lasting for ultralong timescales. Next to high-precision atom interferometric tests, we anticipate that our method will be important for, e. g., shaping a BEC to analyze the wave fronts of light fields and to estimate their possible biases in light-pulse atom interferometers [29].

Obviously, our method can also be employed with attractive or tunable interactions [50] and diverging magnetic lenses [51,52]. Interactions are nowadays also exploited to establish nonclassical correlations. Our method might be of interest in this context. Delta kicks start to be explored in squeezing experiments [53,54] and can be an interesting addition to other squeezing schemes [55]. Furthermore, such spin-polarized, dilute, and slowly expanding gases are important for metrology [56,57]; as well as quantum gas experiments in drop towers [7,36], fountains [10], and space, as envisioned by the BoseEinstein Condensate and Cold Atom Laboratory (BECCAL) space mission aboard the international spacestation (ISS) [58].

We acknowledge valuable discussions with $\mathrm{R}$. Walser. This work is supported by the German Space Agency (DLR) with funds provided by the Federal Ministry for Economic Affairs and Energy (BMWi) due to an enactment of the German Bundestag under Grant No. DLR 50WM1552-1557 (QUANTUS-V Fallturm), as well as by the Centre for Quantum Engineering and Space-Time 
Research and the Deutsche Forschungsgemeinschaft (German Research Foundation) under Germany's Excellence Strategy-EXC 2123 Quantum FrontiersProject No. 390837967 at Leibniz University Hannover.

*Present address: Department of Physics, Stanford University, Stanford, California 94305, USA.

${ }^{\dagger}$ Present address: Quantum Science and Technology Arcetri INO-CNR, Largo Enrico Fermi 2, I-50125 Firenze, Italy. †rasel@iqo.uni-hannover.de

[1] G. Jansen, Nucl. Instrum. Methods Phys. Res., Sect. A 298, 496 (1990).

[2] W. Ketterle, D. S. Durfee, and D. M. Stamper-Kurn, arXiv: cond-mat/9904034.

[3] A. E. Leanhardt, T. A. Pasquini, M. Saba, A. Schirotzek, Y. Shin, D. Kielpinski, D. E. Pritchard, and W. Ketterle, Science 301, 1513 (2003).

[4] P. Medley, D. M. Weld, H. Miyake, D. E. Pritchard, and W. Ketterle, Phys. Rev. Lett. 106, 195301 (2011).

[5] H. Friedburg, Z. Phys. 130, 493 (1951).

[6] H. Ammann and N. Christensen, Phys. Rev. Lett. 78, 2088 (1997).

[7] H. Müntinga, H. Ahlers, M. Krutzik, A. Wenzlawski, S. Arnold, D. Becker et al., Phys. Rev. Lett. 110, 093602 (2013).

[8] J. G. Kalnins, J. M. Amini, and H. Gould, Phys. Rev. A 72, 043406 (2005)

[9] S. Chu, J. Bjorkholm, A. Ashkin, J. Gordon, and L. Hollberg, Opt. Lett. 11, 73 (1986).

[10] T. Kovachy, J. M. Hogan, A. Sugarbaker, S. M. Dickerson, C. A. Donnelly, C. Overstreet, and M. A. Kasevich, Phys. Rev. Lett. 114, 143004 (2015).

[11] D. S. Jin, J. R. Ensher, M. R. Matthews, C. E. Wieman, and E. A. Cornell, Phys. Rev. Lett. 77, 420 (1996).

[12] M.-O. Mewes, M. R. Andrews, N. J. van Druten, D. M. Kurn, D. S. Durfee, C. G. Townsend, and W. Ketterle, Phys. Rev. Lett. 77, 988 (1996).

[13] A. Bassi, K. Lochan, S. Satin, T. P. Singh, and H. Ulbricht, Rev. Mod. Phys. 85, 471 (2013).

[14] S. Nimmrichter and K. Hornberger, Phys. Rev. Lett. 110, 160403 (2013).

[15] S. Vowe, C. Lämmerzahl, and M. Krutzik, Phys. Rev. A 101, 043617 (2020).

[16] S. S. Szigeti, J. E. Debs, J. J. Hope, N. P. Robins, and J. D. Close, New J. Phys. 14, 023009 (2012).

[17] S. Abend, M. Gebbe, M. Gersemann, H. Ahlers, H. Müntinga, E. Giese et al., Phys. Rev. Lett. 117, 203003 (2016).

[18] H. Ahlers, H. Müntinga, A. Wenzlawski, M. Krutzik, G. Tackmann, S. Abend et al., Phys. Rev. Lett. 116, 173601 (2016).

[19] T. Schuldt, C. Schubert, M. Krutzik, L. G. Bote, N. Gaaloul, J. Hartwig et al., Exp. Astron. 39, 167 (2015).

[20] D. N. Aguilera, H. Ahlers, B. Battelier, A. Bawamia, A. Bertoldi, R. Bondarescu et al., Classical Quantum Gravity 31, 115010 (2014).

[21] J. Williams, S.-w. Chiow, N. Yu, and H. Müller, New J. Phys. 18, 025018 (2016).
[22] J. M. Hogan, D. M. Johnson, S. Dickerson, T. Kovachy, A. Sugarbaker, S.-w. Chiow et al., Gen. Relativ. Gravit. 43, 1953 (2011).

[23] P. W. Graham, J. M. Hogan, M. A. Kasevich, and S. Rajendran, Phys. Rev. Lett. 110, 171102 (2013).

[24] G. Rosi, F. Sorrentino, L. Cacciapuoti, M. Prevedelli, and G. Tino, Nature (London) 510, 518 (2014).

[25] D. S. Weiss, B. C. Young, and S. Chu, Phys. Rev. Lett. 70, 2706 (1993).

[26] A. M. Nobili, Phys. Rev. A 93, 023617 (2016).

[27] S. Loriani, C. Schubert, D. Schlippert, W. Ertmer, F. P. Dos Santos, E. M. Rasel, N. Gaaloul, and P. Wolf, Phys. Rev. D 102, 124043 (2020).

[28] T. Hensel, S. Loriani, C. Schubert, F. Fitzek, S. Abend, H. Ahlers, J. N. Siemß, K. Hammerer, E. M. Rasel, and N. Gaaloul, Eur. Phys. J. D 75, 108 (2021).

[29] R. Karcher, A. Imanaliev, S. Merlet, and F. P. Dos Santos, New J. Phys. 20, 113041 (2018).

[30] A. Louchet-Chauvet, T. Farah, Q. Bodart, A. Clairon, A. Landragin, S. Merlet, and F. P. D. Santos, New J. Phys. 13, 065025 (2011).

[31] N. Heine, J. Matthias, M. Sahelgozin, W. Herr, S. Abend, L. Timmen, J. Müller, and E. M. Rasel, Eur. Phys. J. D 74, 174 (2020).

[32] S. Dimopoulos, P. W. Graham, J. M. Hogan, M. A. Kasevich, and S. Rajendran, Phys. Rev. D 78, 122002 (2008).

[33] B. Canuel, S. Abend, P. Amaro-Seoane, F. Badaracco, Q. Beaufils, A. Bertoldi, K. Bongs, P. Bouyer, C. Braxmaier, W. Chaibi et al., Classical Quantum Gravity 37, 225017 (2020).

[34] C. Schubert, D. Schlippert, S. Abend, E. Giese, A. Roura, W. Schleich, W. Ertmer, and E. Rasel, arXiv:1909.01951.

[35] M. Gebbe, J.-N. Siemß, M. Gersemann, H. Müntinga, S. Herrmann, C. Lämmerzahl, H. Ahlers, N. Gaaloul, C. Schubert, K. Hammerer et al., Nat. Commun. 12, 2544 (2021).

[36] J. Rudolph, W. Herr, C. Grzeschik, T. Sternke, A. Grote, M. Popp, D. Becker, H. Müntinga, H. Ahlers, and A. Peters, New J. Phys. 17, 065001 (2015).

[37] Y. T. Baiborodov, M. S. Ioffe, V. M. Petrov, and R. I. Sobolev, J. Nucl. Energy Part C 5, 409 (1963).

[38] D. E. Pritchard, Phys. Rev. Lett. 51, 1336 (1983).

[39] R. Corgier, S. Amri, W. Herr, H. Ahlers, J. Rudolph, D. Guéry-Odelin, E. M. Rasel, E. Charron, and N. Gaaloul, New J. Phys. 20, 055002 (2018).

[40] M. Loy, Phys. Rev. Lett. 32, 814 (1974).

[41] T. van Zoest, N. Gaaloul, Y. Singh, H. Ahlers, W. Herr, S. T. Seidel et al., Science 328, 1540 (2010).

[42] G. Reinaudi, T. Lahaye, Z. Wang, and D. Guéry-Odelin, Opt. Lett. 32, 3143 (2007).

[43] V. M. Perez-Garcia, H. Michinel, J. I. Cirac, M. Lewenstein, and P. Zoller, Phys. Rev. Lett. 77, 5320 (1996).

[44] V. M. Perez-Garcia, H. Michinel, J. I. Cirac, M. Lewenstein, and P. Zoller, Phys. Rev. A 56, 1424 (1997).

[45] See Supplemental Material at http://link.aps.org/ supplemental/10.1103/PhysRevLett.127.100401 for additional information about the variational approach and the fitting procedure. 
[46] D. M. Stamper-Kurn, H.-J. Miesner, S. Inouye, M. R. Andrews, and W. Ketterle, Phys. Rev. Lett. 81, 500 (1998).

[47] D. Guéry-Odelin and S. Stringari, Phys. Rev. Lett. 83, 4452 (1999).

[48] G. Bismut, B. Pasquiou, E. Maréchal, P. Pedri, L. Vernac, O. Gorceix, and B. Laburthe-Tolra, Phys. Rev. Lett. 105, 040404 (2010).

[49] Y. Castin and R. Dum, Phys. Rev. Lett. 77, 5315 (1996).

[50] R. Corgier, S. Loriani, H. Ahlers, K. Posso-Trujillo, C. Schubert, E. M. Rasel, E. Charron, and N. Gaaloul, New J. Phys. 22, 123008 (2020).

[51] J. P. D'Incao, M. Krutzik, E. Elliott, and J. R. Williams, Phys. Rev. A 95, 012701 (2017).

[52] S. Y. Van De Meerakker, H. L. Bethlem, and G. Meijer, Nat. Phys. 4, 595 (2008).
[53] F. Anders, A. Idel, P. Feldmann, D. Bondarenko, S. Loriani, K. Lange, J. Peise, M. Gersemann, B. Meyer, S. Abend et al., arXiv:2010.15796.

[54] Y. Wu, R. Krishnakumar, J. Martínez-Rincón, B. K. Malia, O. Hosten, and M. A. Kasevich, Phys. Rev. A 102, 012224 (2020).

[55] S. S. Szigeti, S. P. Nolan, J. D. Close, and S. A. Haine, Phys. Rev. Lett. 125, 100402 (2020).

[56] P. Asenbaum, C. Overstreet, T. Kovachy, D. D. Brown, J. M. Hogan, and M. A. Kasevich, Phys. Rev. Lett. 118, 183602 (2017).

[57] P. Asenbaum, C. Overstreet, M. Kim, J. Curti, and M. A. Kasevich, Phys. Rev. Lett. 125, 191101 (2020).

[58] K. Frye, S. Abend, W. Bartosch, A. Bawamia, D. Becker, H. Blume, C. Braxmaier, S.-W. Chiow, M. A. Efremov, W. Ertmer et al., Eur. Phys. J. Quantum Technol. 8, 1 (2021). 